CERN-TH.6842/93

UMN-TH-1130/93

ACT-2/93

CTP-TAMU-11/93

\title{
FLIPPED ANGLES AND PHASES: A SYSTEMATIC STUDY
}

\author{
John Ellis \\ Theoretical Physics Division, CERN \\ CH - 1211 Geneva 23 \\ and \\ Jorge L. Lopez and D.V. Nanopoulos \\ Center for Theoretical Physics, Department of Physics, Texas A\&M University \\ College Station, TX 77843-4242, USA \\ and \\ Astroparticle Physics Group, Houston Advanced Research Center (HARC), \\ The Woodlands, TX 77382, USA \\ and \\ Keith A. Olive \\ School of Physics and Astronomy, University of Minnesota \\ Minneapolis, MN 55455, USA
}

\begin{abstract}
We discuss systematically the fermion mass and mixing matrices in a generic field-theoretical flipped $S U(5)$ model, with particular applications to neutrino and baryon number-changing physics. We demonstrate that the different quark flavour branching ratios in proton decay are related to the Cabibbo-Kobayashi-Maskawa angles, whereas the lepton flavour branching ratios are undetermined. The light neutrino mixing angles observable via oscillation effects are related to the heavy conjugate (right-handed) neutrino mass matrix, which also plays a key role in cosmological baryogenesis. The ratios of neutrino and charged lepton decay modes in baryon decay may also be related to neutrino oscillation parameters. Plausible Ansätze for the generation structure of coupling matrices motivate additional relations between physical observables, and yield a satisfactory baryon asymmetry.
\end{abstract}

CERN-TH.6842/93

UMN-TH-1130/93

ACT-2/93

CTP-TAMU-11/93

March 1993 


\section{Introduction}

One of the most welcoming avenues leading beyond the Standard Model is that leading to Grand Unified Theories (GUTs). By unifying the three known particle gauge interactions within a simple group $G$, one may understand why baryon number is conserved to a good approximation, but not perfectly, and why neutrino masses may be very small, but non-zero. In so doing, GUTs also offer scenaria for cosmological baryogenesis and the nature of hot dark matter. Interest in GUTs has been further whetted by the close consistency of the measured values of the $\mathrm{SU}(3), \mathrm{SU}(2)$ and $\mathrm{U}(1)$ gauge couplings with minimal supersymmetric GUTs [1], which high-precision LEP data have rendered even more impressive [2].

With all these phenomenological motivations, it was natural that string modelbuilders should seek to emulate GUTs. However, it was soon realized that there was a sizeable roadblock to deriving a GUT from string: gauge symmetry breaking and various other phenomenological constraints in GUTs generally require adjoint or larger Higgs representations, and these are not obtainable using conventional model-building technology based on $k=1$ Kac-Moody currents on the world-sheet [3]. Hence the revived interest in an $\mathrm{SU}(5) \times \mathrm{U}(1) \mathrm{GUT}$, flipped $\mathrm{SU}(5)$ [ [ , 5], which only required 5- and 10-dimensional Higgs fields and could be derived from string. Flipped $\mathrm{SU}(5)$ is the closest homage string can pay to the simple GUTs of old.

Naturally, there has been considerable discussion within flipped SU(5) of the "classic" new phenomena that motivated so much work on GUTs, namely baryon decay [6] and neutrino masses [5, 7, 8, 9, 10, 11]. However, there has not been a systematic investigation of all the fermion mass matrices and mixing angles that enter into baryon decay branching ratios and neutrino oscillations, and their possible relations to the Cabibbo-KobayashiMaskawa (CKM) quark mixing matrix and cosmological baryogenesis. In this paper we seek to remedy this lack in the literature, working in the general framework of minimal field-theoretical flipped SU(5), supplemented at the end by some (to us) plausible general hypotheses about mass matrices.

It may serve a useful purpose to recall first some important features of minimal fieldtheoretical flipped SU(5) [5]. The first is that baryon decay via dimension-5 operators is very strongly suppressed, and also dimension-6 Higgs exchanges are presumably negligible compared to the dimension- 6 massive vector boson exchange operators expected to dominate. In minimal $\mathrm{SU}(5)$, it was possible to relate the mixing angles in the corresponding dimension- 6 operators for proton decay to the CKM angles, modulo two additional complex phases that would be difficult to measure [12]. The relation is not so direct in flipped $\mathrm{SU}(5)$, because the flipping of the particle assignments within $\underline{\overline{5}}$ and $\underline{10}$ representations, as well as the assignment of conjugate charged leptons to singlet representations, means the branching ratios into different lepton species are independent of the quark mixing angles. It has recently been realized, on the other hand, that the dominant mechanism for baryogenesis in flipped SU(5) may be the decay of massive conjugate ("right-handed") neutrinos [13], producing a lepton asymmetry which is subsequently reprocessed [14] into a baryon asymmetry by non-perturbative electroweak interactions [15]. This raises the possibility that the cosmological baryon asymmetry might be related to observable parameters in the light neutrino mass and mixing matrices, which could also show up in 
baryon decay.

In this paper, we investigate these possibilities systematically, starting from an adaptation in Section 2 of the analysis of ref. [12] to diagonalize the fermion mass matrices and determine the independent unitary flavour rotation matrices. Next, in Section 3 we apply these results to derive interrelations between observables in conventional weak decays, proton decay, neutrino oscillations and cosmological baryogenesis. Then, in Section 4 we postulate plausible Ansätze for the fermion mass matrices which lead to certain additional quantitative relations between the different flavour rotation matrices and hence observable quantities. Finally, in Section 5 we summarize our conclusions and mention directions for future study.

\section{Flipped Mixing Matrices}

Let us first remind the reader of the various coupling matrices that appear in the minimal field-theoretical version of the flipped SU(5) model. The superpotential that characterizes its Yukawa couplings is [5]

$$
W=\lambda_{1}^{i j} F_{i} F_{j} h+\lambda_{2}^{i j} F_{i} \bar{f}_{j} \bar{h}+\lambda_{3}^{i j} \bar{f}_{i} l_{j}^{c} h+\lambda_{4} H H h+\lambda_{5} \bar{H} \bar{H} \bar{h}+\lambda_{6}^{i a} F_{i} \bar{H} \phi_{a}+\lambda_{7} h \bar{h} \phi_{0}+\mu_{a b} \phi_{a} \phi_{b}
$$

where the $F_{i}, \bar{f}_{i}, l^{c}{ }_{i}(\mathrm{i}=1,2,3)$ are the three generations of $\mathbf{1 0}, \overline{\mathbf{5}}$ and singlet representations of $\mathrm{SU}(5)$ that comprise the light matter particles of the Standard Model, $H$ and $\bar{H}$ are $\mathbf{1 0}$ and $\overline{\mathbf{1 0}}$ Higgs representations, $h$ and $\bar{h}$ are $\mathbf{5}$ and $\overline{\mathbf{5}}$ Higgs representations, and the $\phi_{0}, \phi_{a}(\mathrm{a}=1,2,3)$ are auxiliary singlet fields. The first 3 terms in the superpotential (1) give masses to the charge $2 / 3$ quarks $u_{i}$, charge $-1 / 3$ quarks $d_{i}$ and charged leptons $l_{i}$ respectively, the next two terms split the light Higgs doublets from their heavy colour triplet partners in a natural way, the sixth term provides a large element in the see-saw neutrino mass matrix, the product $\lambda_{7}\left\langle\phi_{0}\right\rangle$ gives the traditional Higgs mixing parameter, and the last term is the auxiliary singlet mass matrix [7].

Our task in this section is to diagonalize the coupling matrices $\lambda_{1,2,3,6}$ and the corresponding mass matrices $m_{u, d, l}$, identifying in the process the needed unitary rotation matrices whose observability we will explore in Section 3. Our analysis of the diagonalizations is modelled on that made for minimal $\mathrm{SU}(5)$ in reference [12]. We start by diagonalizing the $\lambda_{2}$ coupling matrix with the unitary transformations

$$
\bar{f}^{\prime}=\bar{f} U_{u^{c}}^{\dagger} \quad, \quad F^{\prime}=U_{u}^{\dagger} F
$$

which yield

$$
\bar{f} \lambda_{2} F=\bar{f}^{\prime} U_{u^{c}} \lambda_{2} U_{u} F^{\prime}=\bar{f}^{\prime} \lambda_{2}^{D} F^{\prime} \quad: \quad \lambda_{2}^{D}=U_{u^{c}} \lambda_{2} U_{u}
$$

As a result, the $\lambda_{1}$ coupling term becomes

$$
F^{T} \lambda_{1} F=F^{\prime T} \lambda_{1}^{\prime} F^{\prime} \quad: \quad \lambda_{1}^{\prime}=U_{u}^{T} \lambda_{1} U_{u}
$$

We now diagonalize this by a further unitary transformation on the fields $F^{\prime}$ :

$$
\widetilde{F}=U_{4} F^{\prime} \quad: \quad \lambda_{1}^{\prime}=U_{4}^{T} \lambda_{1}^{D} U_{4}
$$


As in ref. [12], it is convenient to separate phase factors in the matrix $U_{4}$ : denoting elements of this matrix by $e^{i \eta_{i j}} u_{i j}$ where $\eta_{i j}$ and $u_{i j}$ are both real, we decompose

$$
U_{4}=U_{5} U U_{6} \quad: \quad U_{5}=\operatorname{diag}\left(e^{i \eta_{i 1}}\right) \quad, U_{6}=\operatorname{diag}\left(e^{i \eta_{1 j}}\right) e^{-i \eta_{11}}
$$

This leads to the representation

$$
\lambda_{1}^{\prime}=U_{6} U^{T} U_{7} \lambda_{1}^{D} U U_{6} \quad: \quad U_{7}=U_{5}^{2}
$$

and we impose a phase convention: $\operatorname{det} U_{7}=1$. We can then absorb the $U_{6}$ phases into the fields

$$
\bar{f}^{\prime \prime}=\bar{f}^{\prime} U_{6}^{*} \quad, \quad F^{\prime \prime}=U_{6} F^{\prime}
$$

leaving the diagonalized matrix $\lambda_{2}{ }^{D}$ unchanged. In this basis, the first term in equation (1) becomes

$$
F^{\prime \prime T} U^{T} U_{7} \lambda_{1}^{D} U F^{\prime \prime}
$$

The charge $-1 / 3$ quark mass matrix may finally be cast in real and diagonal form by the redefinitions

$$
\begin{gathered}
F_{\alpha \beta}^{\prime \prime \prime}=U F_{\alpha \beta}^{\prime \prime} \quad \text { for } 1 \leq \alpha, \beta \leq 4 \\
F_{\alpha \beta}^{I V}=U_{7} F_{\alpha \beta}^{\prime \prime \prime}
\end{gathered}
$$

of relevant components of the $\mathbf{1 0}$ representations $F$.

In contrast to the conventional $\mathrm{SU}(5)$ case discussed in ref. [12], the charged lepton mass matrix is not directly related to the quark mass matrices. Starting from the third term in the superpotential (1) and making the above transformations of Eqs. (2, 8) of the 5 fields $\bar{f}$, we find

$$
\bar{f} \lambda_{3} l^{c}=\bar{f}^{\prime \prime}\left(U_{6} U_{u^{c}} \lambda_{3}\right) l^{c}
$$

which we now diagonalize by the new transformations

$$
\bar{f}_{5}^{\prime \prime \prime}=\bar{f}_{5}^{\prime \prime} U_{l}^{\dagger} \quad: \quad l^{c \prime}=U_{l^{c}}^{\dagger} l^{c}
$$

leading to

$$
\bar{f}_{5}^{\prime \prime \prime} \lambda_{3}^{D} l^{c \prime} \quad: \quad \lambda_{3}^{D}=U_{l} U_{6} U_{u^{c}} \lambda_{3} U_{l^{c}}
$$

for the diagonalized mass matrix.

A further novel feature beyond the analysis of ref. [12] is the diagonalization of the light and heavy neutrino mass matrices. We start by diagonalizing the $\phi$ mass matrix:

$$
\phi^{T} \mu \phi=\phi^{T} \mu^{D} \phi^{\prime} \quad: \quad \phi^{\prime}=U_{\phi}^{\dagger} \phi \quad, \mu^{D}=U_{\phi}^{T} \mu U_{\phi}
$$

which leads to the representation

$$
F^{T} \lambda_{6} \phi=F^{\prime \prime T} \lambda_{6}^{\prime} \phi^{\prime} \quad: \quad \lambda_{6}^{\prime}=U_{6}^{*} U_{u}^{T} \lambda_{6} U_{\phi}
$$

The $\nu^{c}=F_{45}$ mass matrix is of the see-saw form in the $\left(F_{45}^{\prime \prime}, \phi^{\prime}\right)$ basis, and so can be written in the form

$$
\left(F_{45}^{\prime \prime}+\ldots\right)^{T} m_{\nu^{c}}^{\prime}\left(F_{45}^{\prime \prime}+\ldots\right) \quad: m_{\nu^{c}}^{\prime}=\lambda_{6}^{\prime T}\left(\mu^{D}\right)^{-1} \lambda_{6}^{\prime}\langle\bar{V}\rangle^{2}
$$


where $\langle\bar{V}\rangle=\langle\overline{\mathbf{1 0}}\rangle$. We recall that the transformations (10) applied to the coloured components of $F$ in order to diagonalize the quark mass matrices have not been applied to the $\nu^{c}=F_{45}$ components. We now define

$$
F_{45}^{\prime \prime \prime}=U_{\nu^{c}}^{\dagger} F_{45}^{\prime \prime}
$$

in terms of which the $\nu^{c}$ mass matrix is diagonal:

$$
\begin{aligned}
F_{45}^{\prime \prime \prime} T m_{\nu^{c}}^{D} F_{45}^{\prime \prime \prime} \quad: & m_{\nu^{c}}^{D}=U_{\nu^{c}}^{T} m_{\nu^{c}}^{\prime} U_{\nu^{c}}=U_{\nu^{c}}^{T} \lambda_{6}^{\prime T}\left(\mu^{D}\right)^{-1} \lambda_{6}^{\prime}\langle\bar{V}\rangle^{2} U_{\nu^{c}}= \\
& U_{\nu^{c}}^{T}\left(U_{\phi}^{T} \lambda_{6}^{T} U_{u} U_{6}^{*}\right)\left(U_{\phi}^{\dagger} \mu^{-1} U_{\phi}^{*}\right)\left(U_{6}^{*} U_{u}^{T} \lambda_{6} U_{\phi}\right)\langle\bar{V}\rangle^{2} U_{\nu^{c}}
\end{aligned}
$$

The light neutrino mass matrix is also of the see-saw form, this time in the $\left(\nu=\bar{f}_{4}, F_{45}\right)$ basis. We diagonalize it in terms of the diagonalized $\nu^{c}$ masses $m_{\nu^{c}}^{D}(18)$. In terms of the unitary transformations already made:

$$
\bar{f}_{4} \lambda_{2} F_{45}=\bar{f}_{4}^{\prime \prime} \lambda_{2}^{D} U_{\nu^{c}} F_{45}^{\prime \prime \prime}
$$

yielding

$$
\bar{f}_{4}^{\prime \prime} m_{\nu} \bar{f}_{4}^{\prime \prime T} \quad: \quad m_{\nu}=\lambda_{2}^{D} U_{\nu^{c}}\left(m_{\nu^{c}}^{D}\right)^{-1} U_{\nu^{c}}^{T} \lambda_{2}^{D}
$$

which we must diagonalize by the further transformation

$$
\bar{f}_{4}^{\prime \prime \prime}=\bar{f}_{4}^{\prime \prime} U_{\nu}^{\dagger} \quad: \quad m_{\nu}^{D}=U_{\nu}\left[\lambda_{2}^{D} U_{\nu^{c}}\left(m_{\nu^{c}}^{D}\right)^{-1} U_{\nu^{c}}^{T} \lambda_{2}^{D}\right] U_{\nu}^{\dagger}
$$

for the light neutrino mass eigenstates.

After this profusion (confusion?) of diagonalizations and unitary matrices, we take mercy on the reader by summarizing the final mass eigenstates (represented by suffices $F)$ :

$$
\begin{array}{rrr}
u_{F}=\left(U_{6} U_{u}^{\dagger}\right) F_{1 \leq \alpha \leq 3,5} & , & u_{F}^{c}=\bar{f}_{1 \leq \alpha \leq 3}\left(U_{u^{c}}^{\dagger} U_{6}^{*}\right) \\
d_{F}=\left(U U_{6} U_{u}^{\dagger}\right) F_{1 \leq \alpha \leq 3,4} & , & d_{F}^{c}=\left(\begin{array}{l}
\left.U_{7} U U_{6} U_{u}^{\dagger}\right) F_{1 \leq \alpha, \beta \leq 3} \\
l_{F}=\bar{f}_{5}\left(U_{u^{c}}^{\dagger} U_{6}^{*} U_{l}^{\dagger}\right)
\end{array}\right. \\
\nu_{F}=\bar{f}_{4}\left(U_{u^{c}}^{\dagger} U_{6}^{*} U_{\nu}^{\dagger}\right) & , & l_{F}^{c}=U_{l^{c}}^{\dagger} l^{c} \\
\nu_{F}^{c}=\left(U_{\nu^{c}}^{\dagger} U_{6} U_{u}^{\dagger}\right) F_{45}
\end{array}
$$

\section{Specific Processes}

As we mentioned earlier, among the motivations for Grand Unified Theories is the possibility for baryogenesis and small neutrino masses. One of the remarkable features of the flipped SU(5) model is its ability to provide cosmologically-interesting neutrino masses (i.e., for $\nu_{\tau}$ ), while at the same time allowing for observable Mikheyev-SmirnovWolfenstein (MSW) [16] neutrino oscillations and baryogenesis via leptogenesis and subsequent sphaleron reprocessing. All three of these highly desirable features are related to the same neutrino mass matrix [10, 13]. Given the complete decomposition of mass eigenstates in the flipped SU(5) model above, we are now in a position to examine in detail the interrelationships between the various mixing matrices and phases of interest. In particular, we will be interested in identifying the mixing matrix for the MSW mixing and the origin of the necessary CP violating phase for the production of the cosmic lepton 
asymmetry. We will also identify the Cabibbo-Kobayashi-Maskawa (CKM) matrix and its role, as well as those of other matrices of interest in proton decay processes.

We begin with the MSW mixing matrix. MSW solar neutrino mixing will result if there is a mismatch between the neutrino states produced in the charged current interactions and the neutrino mass eigenstates. Therefore, we can define the MSW mixing matrix by

$$
l \bar{\nu}=l_{F} U_{M S W} \bar{\nu}_{F}
$$

Given the previous results for the mass eigenstates $l_{F}$ (24) and $\nu_{F}$ (25) we find quite simply

$$
U_{M S W}=U_{l} U_{\nu}^{\dagger}
$$

Similarly, the CKM matrix is the charged current mismatch in the quark sector,

$$
\bar{u} d=\bar{u}_{F} U_{C K M} d_{F}
$$

Using now the expressions (22) and (23) for the mass eigenstates $u_{F}$ and $d_{F}$ we can write

$$
U_{C K M}=U^{\dagger}
$$

A priori, we see no relationship between the quark and neutrino mixing matrices.

Next we study the effective Lagrangian for baryon decay in the flipped SU(5) model, which has been discussed previously in ref. [17]. As has already been recalled, the dominant mechanism for baryon decay is expected to be dimension- 6 vector boson exchange. Using the analysis of the previous section, as was done for conventional $\mathrm{SU}(5)$ in ref. [12], we find the following massive vector boson couplings:

$$
\begin{gathered}
\mathcal{L}_{X}=\frac{g}{\sqrt{2}} X_{i_{\mu}}^{-}\left[\epsilon^{i j k} \bar{d}_{k_{F}}^{c} U_{7} \gamma^{\mu} P_{L} d_{j_{F}}+\bar{u}_{i_{F}} \gamma^{\mu} P_{R} \nu^{c}\right]+\text { h.c. } \\
\mathcal{L}_{Y}=\frac{g}{\sqrt{2}} Y_{i}^{-}{ }_{\mu}\left[\epsilon^{i j k} \bar{d}_{k_{F}}^{c} U_{7} U \gamma^{\mu} P_{L} u_{j_{F}}+\bar{u}_{i_{F}} \gamma^{\mu} P_{R} l^{c}\right]+\text { h.c. }
\end{gathered}
$$

where we have worked in the mass eigenstate basis for the quarks, denoted by subscripts $F$, the lepton fields are in the $\bar{f}^{\prime \prime}$ basis of the previous section, the colour indices $(i, j, k)$ are noted explicitly, and we have indicated the handednesses of all fermion fields by using the projection operators $P_{L}$ and $P_{R}$. The exchanges of $X$ and $Y$ bosons (assumed as usual to have indistinguishable masses) therefore give rise to the following effective Lagrangian for baryon decay:

$$
\begin{aligned}
\mathcal{L}_{\Delta B \neq 0} & =\frac{g^{2}}{2 M_{X}^{2}}\left[\left(\epsilon^{i j k} \bar{d}_{k_{F}}^{c} U_{7} \gamma^{\mu} P_{L} d_{j_{F}}\right)\left(u_{i_{F}} \gamma_{\mu} P_{L} \nu\right)+\right.\text { h.c. } \\
& \left.+\left(\epsilon^{i j k} \bar{d}_{k_{F}}^{c} U_{7} U \gamma^{\mu} P_{L} u_{j_{F}}\right)\left(u_{i_{F}} \gamma_{\mu} P_{L} l\right)+\text { h.c. }\right]
\end{aligned}
$$

As in the case of conventional SU(5), we see from this expression that two additional $C P$ violating phases appear in the quark parts of the $\Delta B \neq 0$ operators [i.e., from $U_{7}=U_{5}^{2}$ in Eq. (6) and $\operatorname{det} U_{7}=1$ ], beyond the single phase in the CKM matrix: these are in principle measurable via loop diagrams. Since the only quarks of relevance for baryon decay at the 
tree level are $u, d$ and $s$, and not more than one of the latter, we can write the relevant parts of the $\Delta B \neq 0$ Lagrangian (above) as:

$$
\begin{aligned}
\overline{\mathcal{L}}_{\Delta B \neq 0} & =\frac{g^{2}}{2 M_{X}^{2}}\left[\left(\epsilon^{i j k} \bar{d}_{k}^{c} e^{2 i \eta_{11}} \gamma^{\mu} P_{L} d_{j}\right)\left(u_{i} \gamma_{\mu} P_{L} \nu_{1}\right)+\right.\text { h.c. } \\
& \left.+\left(\epsilon^{i j k}\left(\bar{d}_{k}^{c} e^{2 i \eta_{11}} \cos \theta_{c}+\bar{s}_{k}^{c} e^{2 i \eta_{21}} \sin \theta_{c}\right) \gamma^{\mu} P_{L} u_{j}\right)\left(u_{i} \gamma_{\mu} P_{L} l_{1}\right)+\text { h.c. }\right]
\end{aligned}
$$

where we recall that $\nu_{L}$ and $l_{L}$ are not in the mass eigenstate basis, so that

$$
\nu_{L}=\nu_{F} U_{\nu} \quad, l_{L}=l_{F} U_{l}
$$

The phase factors in (32) are not measurable, nor can one distinguish between the different neutrino flavours, so the following are the predictions that can be made on the basis of (32):

$$
\frac{\Gamma\left(B \rightarrow(\Sigma \nu)+\left.X\right|_{\text {strange }}\right)}{\Gamma\left(B \rightarrow(\Sigma \nu)+\left.X\right|_{\text {non-strange }}\right)}=0 \quad, \quad \frac{\Gamma\left(B \rightarrow l^{+}+\left.X\right|_{\text {strange }}\right)}{\Gamma\left(B \rightarrow l^{+}+\left.X\right|_{\text {non-strange }}\right)}=\tan ^{2} \theta_{c} \approx \frac{1}{20}
$$

Comparing decays to neutrinos and charged leptons requires knowledge of specific hadronic matrix elements. Following ref. [17], we find

$$
\begin{array}{r}
\Gamma\left(p \rightarrow e^{+} \pi^{o}\right)=\frac{\cos ^{2} \theta_{c}}{2}\left|U_{l_{11}}\right|^{2} \Gamma\left(p \rightarrow \bar{\nu} \pi^{+}\right)=\cos ^{2} \theta_{c}\left|U_{l_{11}}\right|^{2} \Gamma\left(n \rightarrow \bar{\nu} \pi^{o}\right) \\
\Gamma\left(n \rightarrow e^{+} \pi^{-}\right)=2 \Gamma\left(p \rightarrow e^{+} \pi^{o}\right) \quad, \quad \Gamma\left(n \rightarrow \mu^{+} \pi^{-}\right)=2 \Gamma\left(p \rightarrow \mu^{+} \pi^{o}\right) \\
\Gamma\left(p \rightarrow \mu^{+} \pi^{o}\right)=\frac{\cos ^{2} \theta_{c}}{2}\left|U_{l_{12}}\right|^{2} \Gamma\left(p \rightarrow \bar{\nu} \pi^{+}\right)=\cos ^{2} \theta_{c}\left|U_{l_{12}}\right|^{2} \Gamma\left(n \rightarrow \bar{\nu} \pi^{o}\right)
\end{array}
$$

Thus it is possible in principle to correlate baryon decay branching ratios with the CKM angles, and to measure elements of the charged-lepton mixing matrix $U_{l}$, though not of the neutrino mixing matrix $U_{\nu}$. Moreover, we note that the decay branching ratios above are characteristically different from those in conventional SU(5) [17]. However, it lies beyond the scope of this paper to calculate the total baryon decay rate in flipped $\mathrm{SU}(5)$.

We now examine the mixing matrix and the necessary CP-violating phase which can provide for a net lepton asymmetry, and subsequently a net baryon asymmetry via sphaleron reprocessing [14]. As described in Ref. [13], the lepton asymmetry is produced by the out-of-equilibrium decay of the mass eigenstate $\nu_{F}^{c}=F_{45}^{\prime \prime \prime}$ via the mass term $\bar{f}_{4,5} \lambda_{2} F_{45}$. In terms of mass eigenstates the neutrino mass term becomes

$$
\bar{f}_{4,5}^{\prime \prime \prime} U_{\nu, l} \lambda_{2}^{D} U_{\nu^{c}} F_{45}^{\prime \prime \prime}
$$

It is then natural to introduce the lepton-number-violating coupling

$$
\lambda_{L} \equiv U_{\nu, l} \lambda_{2}^{D} U_{\nu^{c}}
$$

We expect the dominant contribution to the lepton (baryon) asymmetry to be that due to decays of the lightest $\nu^{c}$ mass eigenstates, which we expect to be that associated with $\nu_{e, \mu}$ and call $\nu_{1,2}$. The CP asymmetry in the decay of $\nu_{1}^{c}$ into 2 nd and 3rd generation particles is given by

$$
\epsilon_{1}=\frac{1}{2 \pi\left(\lambda_{L}^{\dagger} \lambda_{L}\right)_{11}} \sum_{j}\left(\operatorname{Im}\left[\left(\lambda_{L}^{\dagger} \lambda_{L}\right)_{1 j}\right]^{2}\right) g\left(M_{j}^{2} / M_{1}^{2}\right)
$$


where

$$
g(x)=4 \sqrt{x} \ln \frac{1+x}{x} \quad\left(\simeq \frac{4}{\sqrt{x}} \quad ; x \gg 1\right)
$$

where (38) is the supersymmetric expression for the CP asymmetry [18. Analogous expressions can be written for the corresponding asymmetries $\epsilon_{2,3}$ in the decays of $\nu_{2,3}^{c}$. Using the definition (31) of $\lambda_{L}$ we find

$$
\lambda_{L}^{\dagger} \lambda_{L}=U_{\nu^{c}}^{\dagger}\left(\lambda_{2}^{D}\right)^{2} U_{\nu^{c}}
$$

This depends only on $U_{\nu^{c}}$ and, what is more, the CP-violating phase we are interested in is a priori unrelated to the CKM phase or MSW mixing. In general, we could expect $\left(\lambda_{L}^{\dagger} \lambda_{L}\right)_{11} \sim\left(\lambda_{233}^{D}\right)^{2}$ (the largest entry in $\lambda_{2}^{D}$ ), and if it were the case that $M_{1} \sim M_{2} \ll M_{3}$ we would estimate

$$
\epsilon \simeq \frac{2 \ln 2}{\pi}\left|\lambda_{2}^{D}{ }_{33}\right|^{2} \delta
$$

where $\delta$ is the phase associated with the imaginary part of $\left(\lambda_{L}^{\dagger} \lambda_{L}\right)_{12}$ in (40). (This is slightly larger than our previous estimate [13].) A satisfactory baryon asymmetry would result for $\delta \gtrsim 10^{-2}$. In the next section we will revise this generic estimate in the light of a specific Ansatz for neutrino masses.

\section{Phenomenological Ansätze}

In this section we propose some plausible forms for the various matrices appearing above, and obtain correlations among the various observables of interest. Clearly, the unitary matrices $U$ are not expected to be equal to the identity matrix. However, experience with the CKM and MSW mixing matrices indicates that they probably should not differ too much from unity either. We therefore write

$$
U_{i}=\mathbf{1}+R_{i}
$$

for all matrices $U_{i}$ defined above, with

$$
R_{i}=\left(\begin{array}{ccc}
0 & \theta_{12}^{i} & 0 \\
-\theta_{12}^{* i} & 0 & \theta_{23}^{i} \\
0 & -\theta_{23}^{* i} & 0
\end{array}\right)
$$

such that $U_{i} U_{i}^{\dagger}=\mathbf{1}$ through order $\theta$, since $R_{i}^{\dagger}=-R_{i}$. By analogy with the CKM matrix, we have neglected the far off-diagonal entries in the matrices $R_{i}$.

For the MSW matrix $U_{M S W}=U_{l} U_{\nu}^{\dagger}$ we have $R_{M S W}=R_{l}+R_{\nu}^{\dagger}=R_{l}-R_{\nu}$ and

$$
\theta_{e \mu}=\theta_{12}^{l}-\theta_{12}^{\nu}, \quad \theta_{\mu \tau}=\theta_{23}^{l}-\theta_{23}^{\nu},
$$

where $\theta_{e \mu, \mu \tau}$ are the usual MSW mixing angles. It is interesting to note that the baryon decay branching fractions in Eq. (35) depend in this approximation on

$$
U_{l_{11}}=1, \quad U_{l_{12}}=\theta_{12}^{l},
$$


giving

$$
\Gamma\left(p \rightarrow e^{+} \pi^{0}\right)=\frac{1}{2} \cos ^{2} \theta_{c} \Gamma\left(p \rightarrow \bar{\nu} \pi^{+}\right)=\cos ^{2} \theta_{c} \Gamma\left(n \rightarrow \bar{\nu} \pi^{0}\right) .
$$

Note that our general Ansätze about mixing angles indicate that the baryon decay branching fractions into muons should be suppressed relative to those into electrons. Also, $R_{\nu}$ remains undetermined this way since only the sum over all branching fractions into neutrino final states can be observed. On the other hand, Eqs. (20,21) allow us to obtain $U_{\nu}$ once $U_{\nu^{c}}$ and $m_{\nu^{c}}^{D}$ are given. Since the latter two appear in the baryogenesis parameter $\epsilon$ (Eqs. 38.40), it is interesting to correlate all these parameters.

Starting from Eq. (21) and writing $U_{\nu}=\mathbf{1}+R_{\nu}$ and $U_{\nu^{c}}=\mathbf{1}+R_{\nu^{c}}$ we obtain

$$
m_{\nu_{i}}=\left(\lambda_{2}^{D}\right)_{i}\left(m_{\nu^{c}}^{D}\right)_{i}^{-1}\left(\lambda_{2}^{D}\right)_{i},
$$

which leads to the previously advocated phenomenologically interesting neutrino mass ratios [10]. Moreover, from $\left(m_{\nu}^{D}\right)_{i j}=0$ for $i \neq j$ we find $R_{\nu}$ in terms of $R_{\nu^{c}}$,

$$
\left(R_{\nu}\right)_{i j}=\frac{-1}{m_{\nu_{j}}-m_{\nu_{i}}}\left(\lambda_{2}^{D}\right)_{i}\left(\lambda_{2}^{D}\right)_{j}\left[\left(R_{\nu^{c}}\right)_{i j} \frac{1}{M_{j}}-\left(R_{\nu^{c}}^{*}\right)_{i j} \frac{1}{M_{i}}\right],
$$

where $M_{i} \equiv\left(m_{\nu^{c}}^{D}\right)_{i i}$. In particular

$$
\theta_{12}^{\nu}=\frac{-\lambda_{u} \lambda_{c}}{m_{\nu_{\mu}}-m_{\nu_{e}}}\left[\theta_{12}^{\nu^{c}} \frac{1}{M_{2}}-\theta_{12}^{* \nu^{c}} \frac{1}{M_{1}}\right] \rightarrow \frac{\lambda_{u}}{\lambda_{c}} \theta_{12}^{* \nu^{c}} \frac{M_{2}}{M_{1}} .
$$

The last expression holds in the limit $M_{2} \gg M_{1}$. Also,

$$
\theta_{23}^{\nu}=\frac{-\lambda_{c} \lambda_{t}}{m_{\nu_{\tau}}-m_{\nu_{\mu}}}\left[\theta_{23}^{\nu^{c}} \frac{1}{M_{3}}-\theta_{23}^{* \nu^{c}} \frac{1}{M_{2}}\right] \rightarrow \frac{\lambda_{c}}{\lambda_{t}} \theta_{23}^{* \nu^{c}} \frac{M_{3}}{M_{2}}
$$

with the final expression valid in the limit $M_{3} \gg M_{2}$.

As is plausible for any matrix with a hierarchy of eigenvalues, such as a see-saw mass matrix, we make the Ansatz

$$
\theta_{12}^{\nu^{c}} \simeq \sqrt{\frac{M_{1}}{M_{2}}}, \quad \theta_{23}^{\nu^{c}} \simeq \sqrt{\frac{M_{2}}{M_{3}}}
$$

which gives

$$
\theta_{12}^{\nu} \simeq \frac{\lambda_{u}}{\lambda_{c}} \sqrt{\frac{M_{2}}{M_{1}}}, \quad \theta_{23}^{\nu} \simeq \frac{\lambda_{c}}{\lambda_{t}} \sqrt{\frac{M_{3}}{M_{2}}} .
$$

If for the moment we neglect the $\theta_{12,23}^{l}$ contributions to the neutrino mixing angles [see Eq. (44)], the prediction for $\theta_{\mu \tau}$ in Eq. (52) is exactly what was proposed in Ref. [10] on purely phenomenological grounds. This relation (with $M_{3} / M_{2} \sim 10$ ) and the ratios of neutrino masses in Eq. (47) have been shown [10] to lead to interestingly observable $\nu_{\mu}-\nu_{\tau}$ oscillations and a $\tau$ neutrino mass large enough to provide an interesting amount of astrophysical hot dark matter. The other relation in Eq. (52) should reproduce the present fits to the solar neutrino data based on the MSW mechanism. These require 
$\theta_{e \mu}=(3.2-6.1) \times 10^{-2}$ [19 and therefore $M_{2} / M_{1}=64-225$. (Note: the present expression for $\theta_{e \mu}$ differs from that in Ref. [10].)

Turning now to the baryogenesis parameter $\epsilon$, from Eq. (40) we have (to order $\theta$ )

$$
\left(\lambda_{L}^{\dagger} \lambda_{L}\right)_{i j}=\left(\lambda_{2}^{D}\right)_{i j}^{2} \delta_{i j}+\left(R_{\nu^{c}}\right)_{i j}\left[\left(\lambda_{2}^{D}\right)_{i}^{2}-\left(\lambda_{2}^{D}\right)_{j}^{2}\right]
$$

Thus $\operatorname{Im}\left(\lambda_{L}^{\dagger} \lambda_{L}\right)_{i i}^{2}=\operatorname{Im}\left(\lambda_{L}^{\dagger} \lambda_{L}\right)_{13}^{2}=0$, and

$$
\begin{aligned}
& \operatorname{Im}\left(\lambda_{L}^{\dagger} \lambda_{L}\right)_{12}^{2} \simeq \lambda_{c}^{4}\left|\theta_{12}^{\nu^{c}}\right|^{2} \cos 2 \phi_{12} \\
& \operatorname{Im}\left(\lambda_{L}^{\dagger} \lambda_{L}\right)_{23}^{2} \simeq \lambda_{t}^{4}\left|\theta_{23}^{\nu^{c}}\right|^{2} \cos 2 \phi_{23}
\end{aligned}
$$

where $\phi_{i j}=\arg \left(\theta_{i j}^{\nu^{c}}\right)$. Analogously, the denominator factors are given by

$$
\begin{aligned}
& \left(\lambda_{L}^{\dagger} \lambda_{L}\right)_{11} \simeq \lambda_{u}^{2}+\lambda_{c}^{2}\left|\theta_{12}^{\nu^{c}}\right|^{2} \\
& \left(\lambda_{L}^{\dagger} \lambda_{L}\right)_{22} \simeq \lambda_{c}^{2}+\lambda_{t}^{2}\left|\theta_{23}^{\nu^{c}}\right|^{2} \\
& \left(\lambda_{L}^{\dagger} \lambda_{L}\right)_{33} \simeq \lambda_{t}^{2}
\end{aligned}
$$

We finally get

$$
\begin{aligned}
\epsilon_{1} & =\frac{2}{\pi} \lambda_{c}^{2} \frac{M_{1}}{M_{2}} \delta_{12} \\
\epsilon_{2} & =\frac{2}{\pi} \lambda_{t}^{2} \frac{M_{2}}{M_{3}} \delta_{23} \\
\epsilon_{3} & =\frac{4}{\pi} \lambda_{t}^{2} \frac{M_{2}}{M_{3}} \ln \frac{M_{3}}{M_{2}}\left|\theta_{23}^{\nu^{c}}\right|^{2} \delta_{23}
\end{aligned}
$$

where

$$
\delta_{12}=\cos 2 \phi_{12}, \quad \delta_{23}=\cos 2 \phi_{23}
$$

For the decay of the first and lightest generation $\nu_{1}^{c}$, this result is a factor of $\left(\lambda_{c} / \lambda_{t}\right)^{2}\left(\frac{M_{1}}{\ln 2 M_{2}}\right) \sim$ $10^{-6}$ smaller than that obtained in the generic case in Eq. (41) above. However, we see that for the decay of the second generation $\nu_{2}^{c}$ the result is only suppressed by $\left(\frac{M_{2}}{\ln 2 M_{3}}\right) \sim 7$, and is therefore large enough a priori to produce a satisfactory lepton, and hence baryon, asymmetry.

Normally, it is the lightest generation which produces a net asymmetry [20]. This is because the lightest generation will typically wipe out any prior asymmetries (e.g., via inverse decays) before producing its own. If we considered a standard out-of-equilibrium decay mechanism, we would have to require that both first- and second- generation $\nu^{c}$ 's be far out-of-equilibrium at the time of their decay. This would impose the restrictions $\left(\lambda_{L}^{\dagger} \lambda_{L}\right)_{11,22} \lesssim 10^{3} M_{1,2} / M_{P}$, conditions which would be difficult to satisfy given our Ansätze.

However, invoking a mechanism first proposed in Ref. 21] we assume here that the $\nu^{c}$ 's are produced in the decay of the inflaton, $\eta$, subsequent to inflation. Then $\nu^{c}$ decays occur immediately and out-of-equilibrium at $T \ll M_{\nu^{c}}$, and no destruction of an asymmetry can occur. Thus our only requirement is that $M_{1,2}<m_{\eta}$. This is satisfied 
for $m_{\eta} \sim f e w \times 10^{11} \mathrm{GeV}$ as inferred from the COBE result [22] on density fluctuations [18, 13]. Our final lepton and hence baryon asymmetry now becomes

$$
\frac{n_{B}}{n_{\gamma}} \sim \frac{n_{L}}{n_{\gamma}} \simeq \frac{1}{\Delta}\left(\frac{m_{\eta}}{M_{P}}\right)^{1 / 2}\left(\epsilon_{1}+\epsilon_{2}\right) \sim 10^{-7} \frac{M_{2}}{M_{3}} \delta_{23} \sim 10^{-8} \delta_{23}
$$

where $\Delta \sim 10^{-3}$ is an entropy dilution factor which accounts for the entropy produced during breaking of $S U(5) \times U(1)$ 23, 13. This is encouragingly large: recall the phase $\delta_{23}$ is not related to the standard (CKM) source of $\mathrm{CP}$ violation, and is not expected to be particularly suppressed. Note that there could be extra sources of entropy which would further suppress the estimate (58), so $\delta_{23}$ may not need to be very small.

\section{Summary and Outlook}

We have made in this paper a systematic study of the mass matrices and mixing angles in the minimal field-theoretical version of flipped $\mathrm{SU}(5)$. We have identified the mixing angles that appear in observable processes such as neutrino oscillations and proton decay, as well as the CKM angles in the charged electroweak interactions, and related them as far as possible in a independent way. We have also discussed a scenario for producing the baryon asymmetry via the out-of-equilibrium decays of heavy singlet conjugate neutrinos, which produce a lepton asymmetry that is subsequently reprocessed into a baryon asymmetry by sphaleron transitions. Additional plausible Ansätze for the mixing angles lead to a number of further relations, and a satisfactory baryon asymmetry.

We were motivated to study flipped SU(5) because it is the only GUT that can be derived from string theory, and it was precisely the baryon- and lepton-number-violating processes discussed above that motivated the derivation of a GUT from string. However, the minimal field-theoretical flipped SU(5) model analyzed above is not what one obtains from string theory. On the one hand, flipped SU(5) models derived from string contain more states, thus complicating the analysis, but on the other hand they can cast light on plausible forms for the mass matrices and mixing angles. Therefore it would be desirable to complement this general analysis with some more model-dependent studies.

We believe that flipped SU(5) can give us many insights into the exciting new era of massive neutrino physics that solar neutrino experiments and models of the formation of large-scale astrophysical structures suggest may be opening up before us.

\section{Acknowledgements}

The work of JLL has been supported by an SSC Fellowship. The work of DVN was supported in part by DOE grant DE-FG05-91-ER-40633 and by a grant from Conoco Inc. The work of KAO was supported in part by DOE grant DE-AC02-83ER-40105, and by a Presidential Young Investigator Award. JLL would like to thank the CERN Theory Division for its kind hospitality while part of this work was carried out. 


\section{REFERENCES}

[1] U. Amaldi et al., Phys.Rev. D36 (1987) 85;

G. Costa et al., Nucl.Phys. B297 (1988) 244.

[2] J. Ellis, S. Kelley and D.V. Nanopoulos, Phys.Lett. B249 (1991) 441.

[3] See, e.g.: J. Ellis, J.L. Lopez and D.V. Nanopoulos, Phys.Lett. B245 (1990) 375;

A.M. Font, L.E. Ibáñez and F. Quevedo, Nucl.Phys. B345 (1990) 389.

[4] S. Barr, Phys.Lett. B112 (1982) 219; Phys.Rev. D40(1989) 2457;

J. Derendinger, J. Kim and D.V. Nanopoulos, Phys.Lett. B139 (1984) 170.

[5] I. Antoniadis, J. Ellis, J. Hagelin and D.V. Nanopoulos, Phys.Lett. B194 (1987) 231.

[6] J. Ellis, J.L. Lopez and D.V. Nanopoulos, Phys.Lett. B252 (1990) 53;

G. Leontaris and K. Tamvakis, Phys.Lett. B260 (1991) 333;

G. Leontaris, Z.Phys. C53 (1992) 287.

[7] I. Antoniadis, J. Ellis, J. Hagelin and D.V. Nanopoulos, Phys.Lett. B208 (1988) 209.

[8] G. Leontaris, Phys.Lett. B207 (1988) 447;

G. Leontaris and D.V. Nanopoulos, Phys.Lett. B212 (1988) 327;

G. Leontaris and K. Tamvakis, Phys.Lett. B224 (1989) 319;

S. Abel, Phys.Lett. B234 (1990) 113;

I. Antoniadis, J. Rizos and K. Tamvakis, Phys.Lett. B279 (1992) 281.

[9] J.L. Lopez and D.V. Nanopoulos, Phys.Lett. B268 (1991) 359.

[10] J. Ellis, J.L. Lopez and D.V. Nanopoulos, Phys.Lett. B292 (1992) 189.

[11] G. Leontaris and J. Vergados, Ioannina preprint IOA-290 (1993).

[12] J. Ellis, M.K. Gaillard and D.V. Nanopoulos, Phys.Lett. B88 (1979) 320.

[13] J. Ellis, D.V. Nanopoulos and K.A. Olive, Phys.Lett. B300 (1993) 121.

[14] M. Fukugita and T. Yanagida, Phys.Lett. B174 (1986) 45.

[15] G. 't Hooft, Phys.Rev.Lett. 37 (1976) 8;

V. Kuzmin, V. Rubakov and M. Shaposhnikov, Phys.Lett. B155 (1985) 36.

[16] L.Wolfenstein, Phys.Rev. D17 (1978) 2369; D20 (1979)2634;

S.P. Mikheyev and A. Yu. Smirnov, Yad.Fiz. 42 (1985) 1441; Nuovo Cimento 9C (1986) 17.

[17] J. Ellis, J.S. Hagelin, S. Kelley and D.V. Nanopoulos, Nucl.Phys. B311 (1988/89) 1.

[18] B. Campbell, S. Davidson, and K. A. Olive, University of Minnesota preprint UMNTH-1114/92 (1992), Nucl.Phys B (in press).

[19] GALlEX Collab., P. Anselmann et al., Phys.Lett. B285 (1992) 390.

[20] J.N. Fry, K.A. Olive and M.S. Turner, Phys.Rev.Lett. 45 (1980) 2074.

[21] D.V. Nanopoulos, K.A. Olive, M. Srednicki and K.A. Tamvakis, Phys.Lett. 123B (1983) 41.

[22] F.L. Wright et al. Ap.J.Lett. 396 (1992) L13.

[23] B. Campbell, J. Ellis, J.Hagelin, D.V. Nanopoulos, and K.A. Olive, Phys.Lett. B197 (1987) 355;

B200(1988) 483; J. Ellis, J.Hagelin, D.V. Nanopoulos, and K.A. Olive, Phys.Lett. B207 (1988) 451. 